\section{- OPEN ACCESS}

\title{
Efficacy of subcutaneous interferon $\beta-1$ a on MRI outcomes in a randomised controlled trial of patients with clinically isolated syndromes
}

\author{
Nicola De Stefano, ${ }^{1}$ Giancarlo Comi, ${ }^{2}$ Ludwig Kappos, ${ }^{3}$ Mark S Freedman, ${ }^{4}$ \\ Chris H Polman, ${ }^{5}$ Bernard M J Uitdehaag, ${ }^{5}$ Brian Hennessy, ${ }^{6}$ Florence Casset-Semanaz, ${ }^{7}$ \\ Lorenz Lehr, ${ }^{6}$ Bettina Stubinski, ${ }^{6}$ Dominic L Jack, ${ }^{8}$ Frederik Barkhof ${ }^{9}$
}

\begin{abstract}
- Additional material is published online only. To view please visit the journal online (http://dx.doi.org/10.1136/ jnnp-2013-306289).

${ }^{1}$ Department of Neurological \& Behavioral Sciences, University of Siena, Siena, Italy ${ }^{2}$ Department of Neurology, Ospedale San Raffaele, Milan, Italy

${ }^{3}$ Departments of Neurology and Biomedicine, University Hospital Basel, Basel,

Switzerland

${ }^{4}$ University of Ottawa, Ottawa, Ontario, Canada

${ }^{5}$ VU University Medical Center Amsterdam, Netherlands

${ }^{6}$ Merck Serono SA, Geneva, Switzerland

${ }^{7}$ EMD Serono, Inc., Billerica, Massachusetts, USA

${ }^{8}$ Caudex Medical, Oxford, UK ${ }^{9}$ Diagnostic Radiology and Image Analysis Center (IAC), VU University Medical Center, Amsterdam, Netherlands
\end{abstract}

\section{Correspondence to} Dr Nicola De Stefano, Department of Neurological \& Behavioral Sciences, University of Siena, Viale Bracci 2,

Siena 53100, Italy; destefano@unisi.it

$\mathrm{BH}, \mathrm{LL}$ and $\mathrm{BS}$ affiliation at the time of study.

Received 10 July 2013 Revised 1 November 2013 Accepted 5 November 2013 Published Online First 29 November 2013

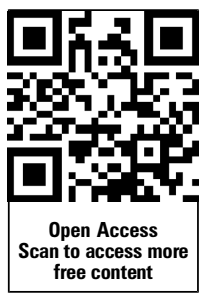

To cite: De Stefano N Comi G, Kappos L, et al. J Neurol Neurosurg Psychiatry 2014;85:647-653.

\section{ABSTRACT}

Aim The REbif FLEXible dosing in early MS (REFLEX) study compared several brain MRI outcomes in patients presenting with clinically isolated syndromes suggestive of multiple sclerosis and treated with two dosefrequencies of subcutaneous interferon (IFN) $\beta$-1a or placebo.

Methods Patients were randomised $(1: 1: 1)$ to IFN $\beta$-1a, $44 \mu \mathrm{g}$ subcutaneously three times a week or once a week, or placebo three times a week for up to 24 months. MRI scans were performed every 3 months, or every 6 months if the patient developed clinically definite multiple sclerosis. End points analysed included: number of combined unique active lesions per patient per scan; numbers and volumes of new $\mathrm{T} 2, \mathrm{~T} 1$ hypointense and gadolinium-enhancing $(\mathrm{Gd}+)$ lesions per patient per scan; and brain volume.

Results 517 patients were randomised (intent-to-treat population: subcutaneous IFN $\beta$-1a three times a week, $n=171$; subcutaneous IFN $\beta$-1a once a week, $n=175$; placebo, $n=171)$. Combined unique active lesions were lower in patients treated with subcutaneous IFN $\beta$-1a versus placebo (mean (SD) lesions per patient per scan: three times a week 0.6 (1.15); once a week 1.23 (4.26); placebo 2.70 (5.23); reduction versus placebo: three times a week $81 \%$; once a week $63 \%$; $p<0.001)$ and with three times a week versus once a week $(48 \%$ reduction; $p=0.002$ ). The mean numbers of new $T 2, T 1$ hypointense and $\mathrm{Gd}+$ lesions were all significantly lower in the two active treatment arms compared with placebo ( $p \leq 0.004$ for three times a week or once a week) and in the three times a week group compared with once a week ( $p \leq 0.012$ ).

Conclusions Both subcutaneous IFN $\beta$ - $1 \mathrm{a} 44 \mu \mathrm{g}$ regimens improved MRI outcomes versus placebo, with the three times a week regimen having a more pronounced effect than once a week dosing. Trial registration clinicaltrial.gov identifier, NCT00404352.

\section{INTRODUCTION}

The first clinical sign or symptom of multiple sclerosis (MS) is often a single neurological event consistent with one or more white matter lesions in the central nervous system, ${ }^{1}$ which is usually followed by the characteristic recurrent attacks of relapsing MS. $^{2}{ }^{3}$ Previous studies have shown that treatment with interferon (IFN) $\beta$ and glatiramer acetate in patients with clinically isolated syndromes (CIS) delays the time to conversion to clinically definite MS (CDMS). ${ }^{4-7}$ Most recently, the REFLEX (REbif FLEXible dosing in early MS) trial found that IFN $\beta-1 \mathrm{a}, 44 \mu \mathrm{g}$ subcutaneously three times a week or once a week, reduced the risk of conversion from CIS to MS over 24 months. $^{8}$

The primary end point in the REFLEX study was the onset of MS defined by the 'McDonald' 2005 criteria. ${ }^{8}$ The McDonald criteria have allowed the use of MRI to help determine whether central nervous system lesions are disseminated in space and time. ${ }^{9-11}$

The study demonstrated a significant difference between the two different dose-frequencies of subcutaneous IFN $\beta-1 \mathrm{a}$ on time to McDonald 2005 MS, but no significant difference in time to CDMS. ${ }^{8}$ This difference was likely detected because of the increased sensitivity of MS diagnosis aided by MRI, as lesions may occur more frequently than relapses, ${ }^{12} 13$ and the larger proportion of patients who converted to McDonald 2005 MS compared with CDMS. Here we present the detailed MRI results from the REFLEX trial that may underpin the clinical observations.

\section{METHODS}

Standard protocol approvals, registrations and patient consents

The REFLEX trial (ClinicalTrials.gov identifier: NCT00404352) was conducted in compliance with the Declaration of Helsinki and standards of Good Clinical Practice according to the International Conference on Harmonisation of Technical Requirements for Registration of Pharmaceuticals for Human Use. The institutional review boards of all participating centres approved the study protocol; all patients provided written informed consent before entering into the trial.

\section{Patients and procedures}

The design of the REFLEX trial has been described in detail elsewhere. ${ }^{8}$ Briefly, eligible patients were aged 18-50 years with an Expanded Disability Status Scale score of 0.0-5.0, had experienced a single demyelinating event suggestive of MS within 60 days prior to study entry and had $\geq 2$ clinically silent lesions on a T2-weighted brain MRI scan with a size of at least $3 \mathrm{~mm}$, at least one of which is 
ovoid or periventricular or infratentorial. Based on these characteristics, these patients were considered to be at high risk of conversion to MS. ${ }^{14}$ Patients were randomised $(1: 1: 1)$ to receive the serum-free formulation of IFN $\beta-1 \mathrm{a}, 44 \mu \mathrm{g}$ subcutaneously three times a week or once a week, or placebo for 24 months or until conversion to CDMS, as defined by either a second event suggestive of MS or a 3-month sustained increase ( $\geq 1.5$ points) in Expanded Disability Status Scale score. The double blind (DB) period was defined as the interval between randomisation and conversion to CDMS, or study discontinuation, or 24 months, whichever occurred first. The primary end point of the study was time to McDonald MS (2005 criteria), ${ }^{9}$ and has previously been reported. ${ }^{8}$

\section{MRI assessments}

MRI assessments were made every 3 months from baseline to Month 24. For patients who converted to CDMS before Month 24 , MRI scans were subsequently performed at 6-month intervals at predefined time points with respect to the baseline MRI (at Month 6, Month 12, Month 18 and Month 24). The same MRI machine at each centre was used for all scans for each patient throughout the study. Analysis and evaluation of MRI scans were performed centrally at the VU University Medical Center, Amsterdam, The Netherlands.

The main MRI end point of the study was the mean number of combined unique active (CUA) lesions per patient per scan (main secondary endpoint). A CUA lesion was defined as a new or persisting gadolinium-enhancing $(\mathrm{Gd}+)$ lesion on T1 MRI or a new or enlarging lesion on T2 MRI (non-enhancing on T1 MRI), and lesions identified in both scans were only counted once. Additional secondary end points described in this paper were: mean number of new non-enhancing T2 lesions per patient per scan, mean number of new $\mathrm{Gd}+$ lesions per patient per scan, T2 lesion volume, mean number of new T1 hypointense (non-enhancing) lesions per patient per scan, T1 hypointense (non-enhancing) lesion volume, T1 Gd+ lesion volume, and brain volume determined at yearly intervals. The effect of treatment on the percentage change in brain volume from baseline was assessed using the Structural Image Evaluation using Normalisation of Atrophy method. ${ }^{14}$

\section{Clinical assessments}

Clinical assessments, including the monitoring of relapses, have been described previously. ${ }^{8}$

\section{Statistical analyses}

Efficacy analyses were performed on the intent-to-treat (ITT) population, which consisted of all randomised patients, analysed by their original allocation.

Analysis of CUA lesions per patient per scan was part of the hierarchical statistical testing process described previously, ${ }^{8}$ and consisted of the pairwise comparison of the three treatment groups at the 0.05 significance level using a non-parametric analysis of variance on ranks model, including treatment and randomisation stratification factors as covariates. The main MRI-based secondary end point analysed in the confirmatory analysis was the mean number of CUA lesions per patient per scan from randomisation to Month 24 or conversion to CDMS, whichever occurred first (DB period). The mean number of CUA lesions per patient per scan over the whole study period (from randomisation to Month 24, irrespective of conversion to CDMS) was considered an exploratory analysis.

Treatment effect sizes with corresponding 95\% CIs were estimated using an adjusted negative binomial model that included treatment and randomisation factors as covariates. Treatment effects are given as ratios because of the logarithmic structure of the model used. The other MRI end points (T2, T1 hypointense and Gd+ lesions) were analysed in the same manner as CUA lesions. Brain and lesion volume were reported using descriptive statistics.

The patient baseline subgroups analysed were all predefined and comprised those used to stratify the randomisation process (age $<30$ years or $\geq 30$ years, steroid use or not at first attack, monofocal/multifocal presentation of first attack, presence or absence of $\mathrm{Gd}+$ lesions at baseline) and other subgroups of interest (sex, $<9$ or $\geq 9 \mathrm{~T} 2$ lesions at baseline). A post hoc analysis was performed to test the treatment-by-subgroup interactions. A negative binomial model was fitted with treatment, each of the six subgroups and the six treatment-by-subgroup interactions. Backward elimination was used to select the model with the best fit.

\section{RESULTS}

Patients, conversion to MS and safety

A total of 701 patients were screened and 517 were randomised (ITT population: subcutaneous IFN $\beta$ - 1 a three times a week, $\mathrm{n}=171$; subcutaneous IFN $\beta$-1a once a week, $\mathrm{n}=175$; placebo, $\mathrm{n}=171) ; 515$ patients received the study drug or placebo. The disposition of patients has been described in detail elsewhere. ${ }^{8}$ The groups showed similar baseline demographics, disease and MRI characteristics (table 1).

In the IFN $\beta$-1a three times a week, once a week and placebo groups, 146 (85.4\%), 156 (89.1\%) and 146 (85.4\%) patients, respectively, completed the whole treatment period. Reasons given for treatment discontinuation have been described previously. ${ }^{8}$ Results for conversion to MS have been described previously $^{8}$ (see online supplementary table S1). No new or unexpected safety signals were observed during the study. ${ }^{8}$

\section{Lesions per patient per scan}

The mean (SD) number of CUA lesions per patient per scan in the ITT population during the DB treatment period was 0.6 (1.15) in the three times a week group, 1.23 (4.26) in the once a week group and 2.70 (5.23) in the placebo group, corresponding to reductions versus placebo of 2.10 and 1.47 lesions per patient per scan for three times a week and once a week, respectively $(p<0.001$ for three times a week and once a week vs placebo; $p=0.002$ for three times a week vs once a week; two-sided stratified non-parametric analysis of variance). ${ }^{8}$ At 3 months, mean CUA lesions per patient per scan in the DB period were lower in patients treated with IFN $\beta$-1a than at baseline, with a further reduction at 6 months (figure 1). The mean CUA lesions per patient per scan reduced over time in placebo-treated patients during the DB period.

An analysis using an adjusted negative binomial model showed a reduction in the mean number of CUA lesions, with risk reductions versus placebo of $81 \%$ for three times a week and $63 \%$ for once a week (table 2). The mean number of CUA lesions per patient per scan was $48 \%$ lower in the three times a week group than in the once a week group (reduction of 0.45 CUA lesions per patient per scan).

In the DB period, the mean numbers of new T2, T1 hypointense and $\mathrm{Gd}+$ lesions per patient per scan were all significantly lower in the two active treatment arms compared with placebo ( $p \leq 0.004$ for three times a week and once a week vs placebo, all lesions; table 2). Compared with placebo, with subcutaneous IFN $\beta-1$ a there was a $57-70 \%$ reduction in new T2 lesions, $37-57 \%$ reduction in $\mathrm{T} 1$ hypointense lesions and $76-92 \%$ 
Table 1 Baseline demographics and MRI characteristics (intent-to-treat population)

\begin{tabular}{|c|c|c|c|c|}
\hline Characteristic & $\begin{array}{l}\text { IFN } \beta-1 \mathrm{a} \text {, } \\
44 \mu \mathrm{g} \text { subcutaneously } \\
\text { three times a week } \\
(\mathrm{n}=171)\end{array}$ & $\begin{array}{l}\text { IFN } \beta-1 \mathrm{a} \text {, } \\
44 \mu \mathrm{g} \text { subcutaneously } \\
\text { once a week } \\
(\mathrm{n}=175)\end{array}$ & $\begin{array}{l}\text { Placebo } \\
(n=171)\end{array}$ & $\begin{array}{l}\text { Overall } \\
(\mathrm{N}=517)\end{array}$ \\
\hline \multicolumn{5}{|l|}{ Age, years } \\
\hline Mean (SD) & $30.6(8.5)$ & $30.7(8.1)$ & $30.9(7.9)$ & $30.7(8.2)$ \\
\hline Median (Q1, Q3) & $29.0(24.0,36.0)$ & $30.0(25.0,37.0)$ & $29.0(25.0 .37 .0)$ & $29.0(24.0,37.0)$ \\
\hline Female, n (\%) & $114(66.7)$ & $106(60.6)$ & $112(65.5)$ & $332(64.2)$ \\
\hline $\begin{array}{l}\text { Classification of first clinical demyelinating event } \\
\text { as monofocal, }{ }^{*} \mathrm{n}(\%)\end{array}$ & $96(56.1)$ & $90(51.4)$ & $91(53.2)$ & $277(53.6)$ \\
\hline Steroid use at first clinical demyelinating event, $\mathrm{n}(\%)$ & $121(70.8)$ & $123(70.3)$ & $121(70.8)$ & $365(70.6)$ \\
\hline \multicolumn{5}{|l|}{ EDSS score } \\
\hline Mean (SD) & $1.56(0.81)$ & $1.56(0.74)$ & $1.57(0.76)$ & $1.56(0.77)$ \\
\hline Median (Q1, Q3) & $1.50(1.00,2.00)$ & $1.50(1.00,2.00)$ & $1.50(1.00,2.00)$ & $1.50(1.00,2.00)$ \\
\hline \multicolumn{5}{|l|}{ Gd+ lesions } \\
\hline Presence of at least $1 \mathrm{~T} 1 \mathrm{Gd}+$ lesion, $\mathrm{n}(\%)$ & $68(39.8)$ & $72(41.1)$ & $73(42.7)$ & $213(41.2)$ \\
\hline \multicolumn{5}{|l|}{$\mathrm{T} 1 \mathrm{Gd}+$ lesions } \\
\hline Mean (SD) & $1.3(2.5)$ & $1.5(3.5)$ & $1.2(2.7)$ & $1.3(2.9)$ \\
\hline Median (Q1, Q3) & $0.0(0.0,2.0)$ & $0.0(0.0,1.0)$ & $0.0(0.0,1.0)$ & $0.0(0.0,1.0)$ \\
\hline \multicolumn{5}{|l|}{$\mathrm{T} 1 \mathrm{Gd}+$ lesion volume, $\mathrm{mm}^{3}$} \\
\hline Mean (SD) & $156.54(427.33)$ & $194.15(593.66)$ & $193.68(588.50)$ & $181.56(541.68)$ \\
\hline Median (Q1, Q3) & $0.00(0.00,91.60)$ & $0.00(0.00,91.60)$ & $0.00(0.00,120.20)$ & $0.00(0.00,94.40)$ \\
\hline \multicolumn{5}{|l|}{ T1 hypointense lesions } \\
\hline Mean (SD) & $5.7(6.8)$ & $5.9(7.5)$ & $5.7(8.0)$ & $5.8(7.4)$ \\
\hline Median (Q1, Q3) & $4.0(1.0,8.0)$ & $3.0(1.0,7.0)$ & $3.0(1.0,8.0)$ & $3.0(1.0,8.0)$ \\
\hline \multicolumn{5}{|l|}{$\mathrm{T} 1$ hypointense lesion volume, $\mathrm{mm}^{3}$} \\
\hline Mean (SD) & $675.02(1049.85)$ & $774.80(1287.98)$ & $670.29(1054.13)$ & $707.23(1136.20)$ \\
\hline Median (Q1, Q3) & $280.40(51.50,984.20)$ & $203.10(42.90,895.50)$ & $266.10(17.10,892.60)$ & $231.70(40.10,893.70)$ \\
\hline \multicolumn{5}{|l|}{ T2 lesions } \\
\hline Mean (SD) & $22.0(18.8)$ & $23.6(21.0)$ & $21.3(20.2)$ & $22.3(20.0)$ \\
\hline Median (Q1, Q3) & $17.0(9.0,29.0)$ & $17.0(8.0,35.0)$ & $15.0(7.0,28.0)$ & $17.0(8.0,30.0)$ \\
\hline$\geq 9$ T2 lesions, $n(\%)$ & $129(75.4)$ & $126(72.0)$ & $122(71.3)$ & 377 (72.9) \\
\hline \multicolumn{5}{|l|}{$\mathrm{T} 2$ lesion volume, $\mathrm{mm}^{3}$} \\
\hline Mean (SD) & $3110.53(310.74)$ & $3853.12(4716.71)$ & $3334.92(3990.41)$ & $3436.11(4083.90)$ \\
\hline Median (Q1, Q3) & $2039.90(755.30,3876.70)$ & $2057.00(675.20,5722.20)$ & $1778.30(695.20,4208.50)$ & $1928.40(709.50,4240.00)$ \\
\hline \multicolumn{5}{|l|}{ Normalised brain volume, $\mathrm{cm}^{3}$} \\
\hline Mean (SD) & $1536.2(73.69)$ & $1537.3(66.47)$ & $1543.6(65.64)$ & $1539.0(68.63)$ \\
\hline Median (Q1, Q3) & $1538.57(1491.70,1597.36)$ & $1539.97(1500.81,1574.96)$ & $1547.60(1497.16,1589.08)$ & $1544.10(1497.16,1586.13)$ \\
\hline
\end{tabular}

Figure 1 Mean number of CUA lesions during the double blind period (intent-to-treat population). $p$ Values derived from pairwise non-parametric analysis of variance. CUA, combined unique active; IFN, interferon; Sc, subcutaneous; tiw, three times a week; qw, once a week.

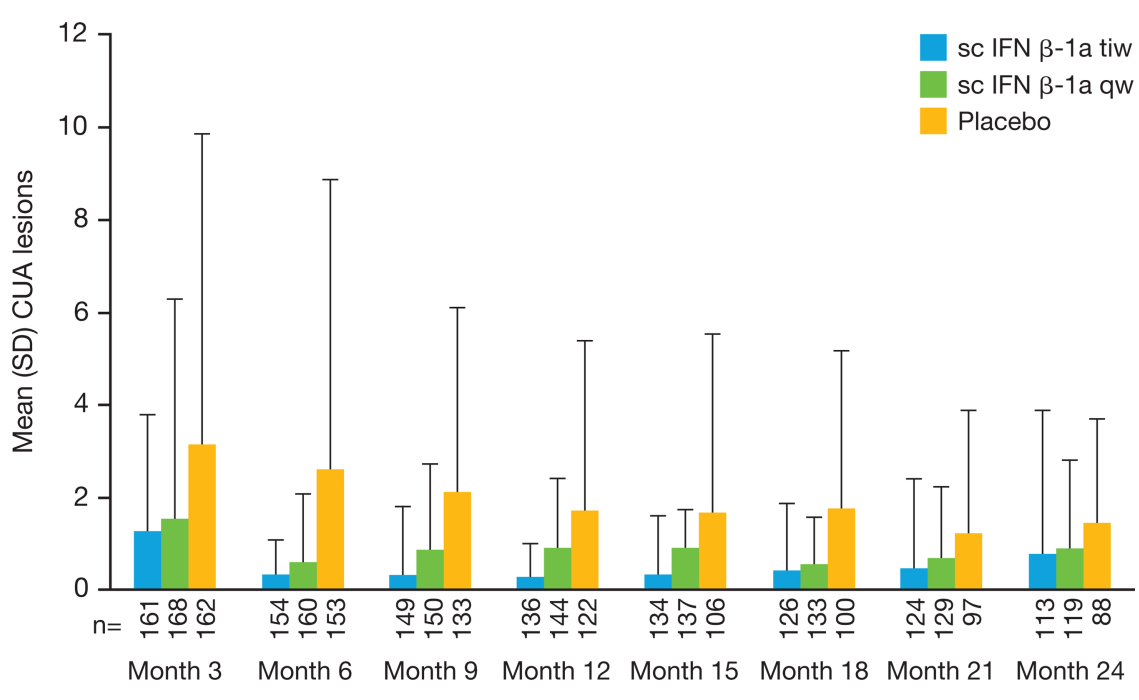




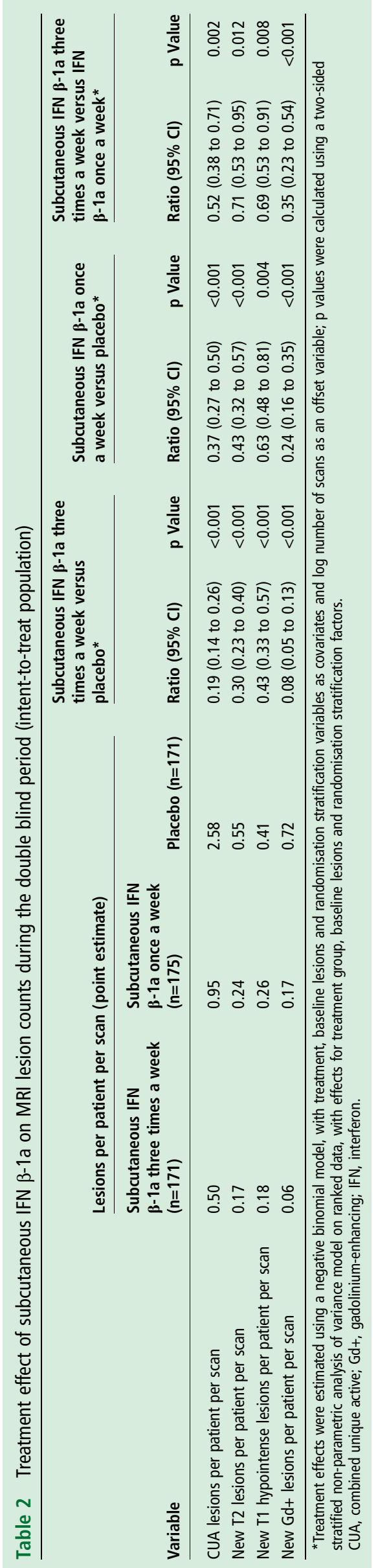

reduction in Gd+ lesions. There was a significant difference between the three times a week and once a week groups for all three end points $(p \leq 0.012)$, with reductions of up to $65 \%$ in new MRI lesions in the three times a week versus once a week group. Similarly, the proportion of patients free from new lesions during the DB period was higher in the three times a week group than in the placebo or once a week groups (table 3). Over the whole study period, the patterns of numbers of lesions per patient per scan were similar to that observed in the DB period (see online supplementary figure S1), despite the higher number of patients in the placebo group who converted to CDMS and thereafter received IFN $\beta-1 \mathrm{a}, 44 \mu \mathrm{g}$ subcutaneously three times a week, during the 2 years of the trial, compared with the active treatment groups.

\section{Treatment effect on CUA lesions, by patient baseline subgroups}

The baseline subgroup variables age, presence of $\mathrm{Gd}+$ lesions and number of T2 lesions were significant baseline factors in the accumulation of CUA lesions per patient per scan during the DB period: age (HR for age $<30$ years vs $\geq 30$ years: $1.69,95 \%$ CI 1.31 to 2.18 ), presence of $\mathrm{Gd}+$ lesions ( $\mathrm{HR}$ for $\geq 1$ lesion vs 0 lesions: $2.67,95 \%$ CI 2.05 to 3.47 ) and number of T2 lesions (HR for $\geq 9$ lesions vs $<9$ lesions: $4.93,95 \%$ CI 3.66 to 6.63 ). There was no significant effect of monofocal versus multifocal presentation, use of steroids during the first event or patient sex.

During the DB period, the least squares mean ratios of CUA lesions for three times a week versus placebo, once a week versus placebo and three times a week versus once a week in the prespecified subgroups were broadly similar to those obtained in the ITT population (see online supplementary table S2). There was a significant treatment effect versus placebo in all the prespecified subgroups treated three times a week and once a week compared with placebo, except male patients treated once a week. A statistically significant quantitative treatment-by-sex interaction was observed $(p=0.003)$, with the treatment effect in male patients lower than that in female patients for three times a week and once a week compared with placebo. However, the treatment effect was still positive for men in both doses versus placebo. No other treatment-by-subgroup interactions were observed; treatment effects were similar between patients with greater or lesser disease activity at baseline, as determined by the number of $\mathrm{Gd}+$ or $\mathrm{T} 2$ lesions, monofocal versus multifocal presentation or steroid use during the CIS (data not shown). There was a significant difference between the three times a week and once a week groups in most of the prespecified subgroups during the DB period (see online supplementary table S2).

\section{MRI lesion volume and brain volume}

There was an indication of a dose-dependent effect of subcutaneous IFN $\beta-1 \mathrm{a}$ on the change in the last observed value (LOV) of T2 lesion volume during the DB period (see online supplementary figure S1). Median (IQR) T2 lesion volume decreased from baseline in the three times a week group by $-128.7 \mathrm{~mm}^{3}\left(-721.0 \mathrm{~mm}^{3}\right.$ to $\left.42.5 \mathrm{~mm}^{3}\right)$ and by $-37.9 \mathrm{~mm}^{3}$ $\left(-609.3 \mathrm{~mm}^{3}\right.$ to $\left.177.4 \mathrm{~mm}^{3}\right)$ in the once a week group, but increased by $51.5 \mathrm{~mm}^{3}\left(-194.6 \mathrm{~mm}^{3}\right.$ to $\left.617.3 \mathrm{~mm}^{3}\right)$ in the placebo group. Median (IQR) change from baseline in T1-Gd+ lesion volume was $0 \mathrm{~mm}^{3}\left(-88.7 \mathrm{~mm}^{3}\right.$ to $\left.0.0 \mathrm{~mm}^{3}\right)$ in the three times a week group, $0 \mathrm{~mm}^{3}\left(-71.55 \mathrm{~mm}^{3}\right.$ to $\left.0.00 \mathrm{~mm}^{3}\right)$ in the once a week group and $0 \mathrm{~mm}^{3}\left(-54.40 \mathrm{~mm}^{3}\right.$ to $\left.11.40 \mathrm{~mm}^{3}\right)$ in the placebo group. Median T1 hypointense lesion volume did not change from baseline in the three times a week and once a 
Table 3 Percentage of patients with no new lesions during the double blind period (intent-to-treat population)

\begin{tabular}{lccc}
\hline & Proportion of patients free from new lesions, $\mathbf{n}(\%)$ & & \\
\cline { 2 - 3 } Variable & Subcutaneous IFN $\boldsymbol{\beta}$-1a three times a week $(\mathbf{n}=\mathbf{1 7 1 )}$ & Subcutaneous IFN $\boldsymbol{\beta}$-1a once a week (n=175) & Placebo ( $\mathbf{n = 1 7 1 )}$ \\
\hline New T2 lesions & $65(40.1)$ & $46(27.4)$ & $32(19.8)$ \\
New T1 hypointense lesions & $67(41.4)$ & $45(26.8)$ & $40(24.7)$ \\
New Gd+ lesions & $120(74.1)$ & $84(50.0)$ & $49(30.2)$ \\
New Gd+ or new T2 lesions & $59(36.4)$ & $37(22.0)$ & $24(14.8)$ \\
\hline
\end{tabular}

$\mathrm{Gd}+$, gadolinium-enhancing; IFN, interferon.

week groups, while in the placebo group there was an increase of $31.5 \mathrm{~mm}^{3}$ from baseline. Results for median $\mathrm{T} 2$ and $\mathrm{T} 1$ hypointense lesion volumes in the whole study period were similar to those observed in the DB study period (data not shown).

During the DB period, changes in brain volume from baseline at Month 12 and Month 24, and the LOV, were similar across the different treatment groups (range $-0.34 \%$ to $-1.04 \%$; figure 2), although the greatest loss was in the three times a week group compared with the once a week and placebo groups. Observations over the whole study period were similar.

\section{DISCUSSION}

The previously reported results of the REFLEX trial demonstrated the clinical benefits of treatment with subcutaneous IFN $\beta-1 \mathrm{a}$ initiated at presentation of CIS. The MRI findings reported here further support those results. Treatment with subcutaneous IFN $\beta-1 \mathrm{a}$ at both dosing frequencies significantly reduced the number of CUA lesions compared with placebo, which likely contributed to the treatment effect observed on conversion to McDonald MS already described. ${ }^{8}$ Importantly, a clear dose effect was observed, with a significantly greater reduction in the number of CUA lesions with the three times a week dose
Figure 2 Percentage change in brain volume from baseline, by visit (intent-to-treat population), during the (A) double blind period and (B) whole study period. Red lines represent the median, boxes represent the IQR, and whiskers represent the range. IFN, interferon; LOV, last observed value; sc, subcutaneously; tiw, three times a week; qw, once a week.

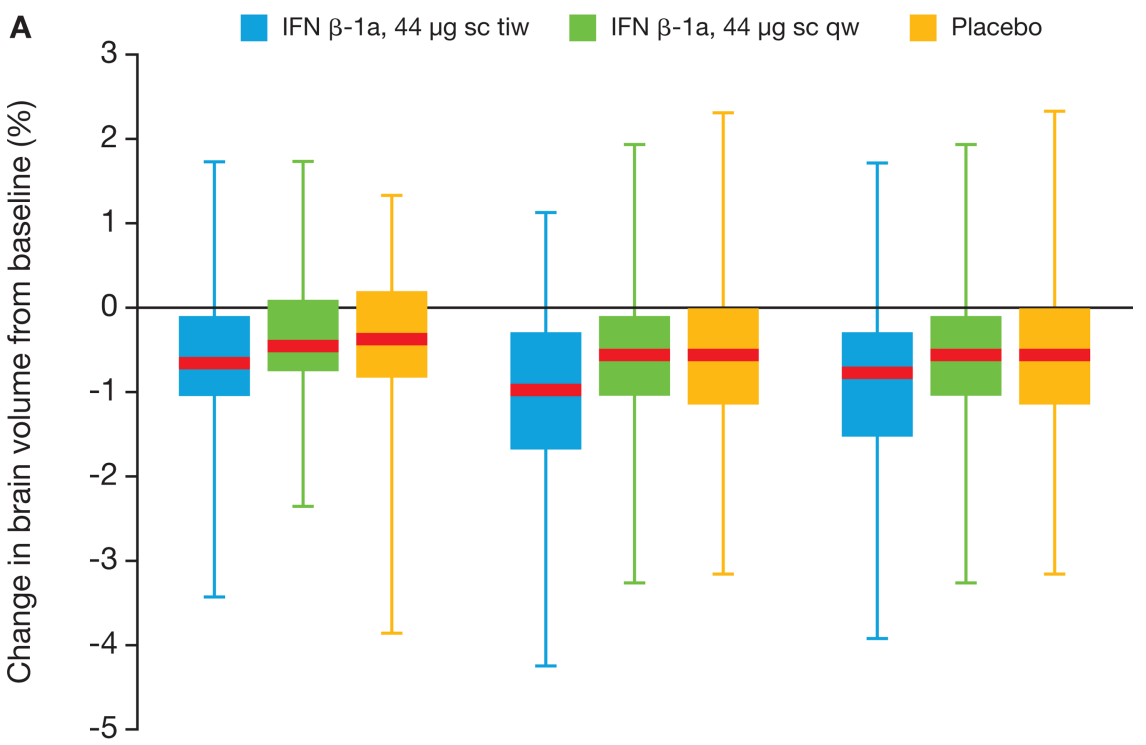

B

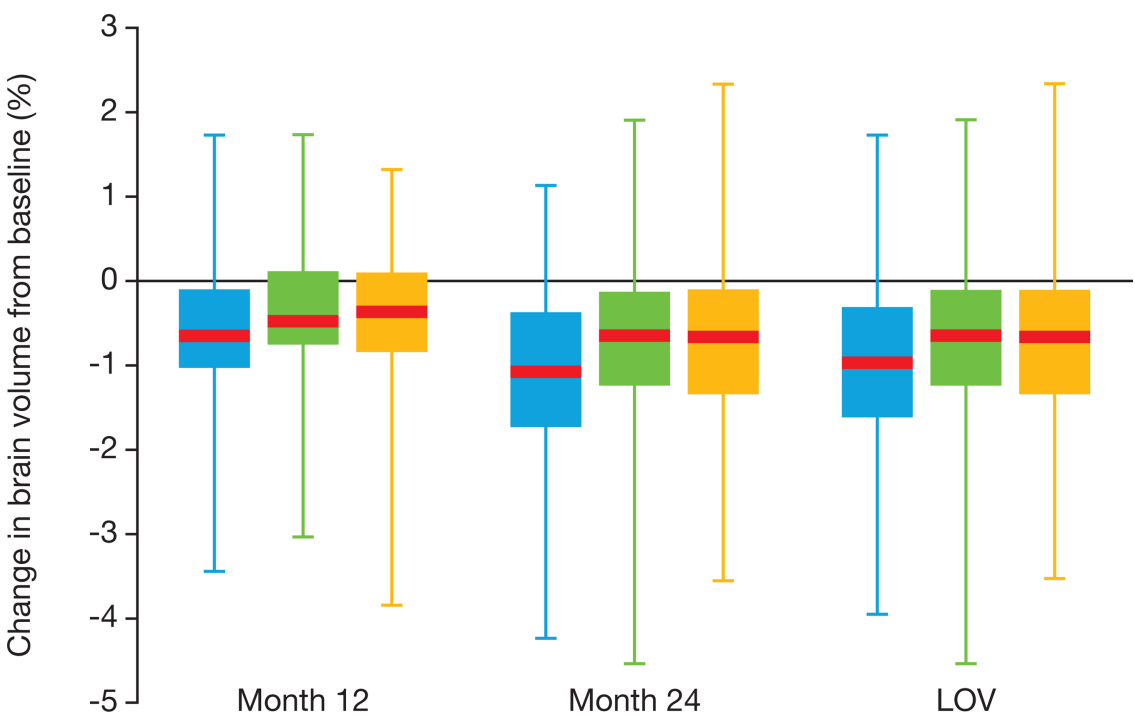


regimen compared with the once a week dose regimen. These results support previous reports of the effect of IFN $\beta$ in patients with CIS. ${ }^{15-17}$ Subcutaneous IFN $\beta-1 b$ and intramuscular IFN $\beta-1$ a have been shown to significantly improve MRI outcomes in patients with CIS at 2 years ${ }^{15}$ or 3 years. ${ }^{17}$ The advantages of early treatment of patients with CIS with IFN $\beta$ versus delayed treatment appear to extend to at least 5 years in terms of MRI outcomes. ${ }^{18} 19$ In the current study the dose effect was robust across lesion subtypes and was observed during the whole study period, including patients originally randomised to the placebo or once a week group who converted to CDMS and who subsequently received open-label IFN $\beta-1 \mathrm{a}$, $44 \mu \mathrm{g}$ subcutaneously three times a week (see online supplementary figure S1).

CUA lesions in all treatment groups reduced over the course of the DB period. Patients who converted to CDMS would no longer be included in the DB period analysis, and would be expected to have more CUA lesions than those who did not convert. In the subcutaneous IFN $\beta$-1a groups, the number of CUA lesions appeared to be higher at 24 months than at 12 months. However, this appears to be an effect of a small number of patients with large numbers of lesions at Month 24 and the reduced number of patients still in the DB analysis compared with baseline.

Previous studies have shown that certain demographic characteristics, MRI findings at baseline and the need for steroid treatment during the CIS have been linked to more rapid progression to, and modified prognosis of, MS. ${ }^{4} 20-23$ These characteristics could potentially affect the efficacy of therapy intended to delay MS. However, in an exploratory analysis, the efficacy of subcutaneous IFN $\beta$ - 1 a three times a week versus placebo in the predefined subgroups in REFLEX was broadly similar to that found in the ITT population. This differs from observations with subcutaneous IFN $\beta-1 b$, in which a lower treatment effect was found in patients with more evidence of disease dissemination. ${ }^{20}$ The dose effect of three times a week and once a week compared with placebo observed in most of the predefined subgroups in the REFLEX study was similar to that observed in the ITT population, except sex where a significant quantitative treatment-by-sex interaction was observed; female patients had a more pronounced treatment effect compared with male patients. This also contrasts with the findings of a previous trial of subcutaneous IFN $\beta-1 b$, which revealed a greater treatment effect in male patients. ${ }^{20}$ Bearing in mind the similarities of these trials and populations, it is difficult to interpret either finding.

Markers of neurodegeneration were measured in the study. During the DB period, there was a dose-dependent beneficial effect of subcutaneous IFN $\beta-1 \mathrm{a}$ on the number and volume of T1 hypointense lesions, which are associated with axonal damage and loss. ${ }^{24}$ In contrast, the percentage brain volume loss in patients in the once a week group was similar to the placebo group, but there appeared to be a greater loss in the three times a week group compared with placebo. In this context, a previous study in patients with CIS had suggested that subcutaneous IFN $\beta$-1a at a lower dose than used in REFLEX resulted in a reduction in the loss of brain volume. ${ }^{25}$ However, studies with glatiramer acetate or subcutaneous IFN $\beta$-1b have also not shown a significant effect on brain volume changes. ${ }^{4} 619$ Indeed, present and previous observations could have been confounded by the effect of pseudoatrophy, which may occur due to the resolution of inflammation with IFN $\beta .^{26}$ It is important to note that, in REFLEX, brain volume was recorded annually and, for patients who converted to CDMS in the first 12 months, the LOV for brain volume was Month 12, regardless of the date of CDMS conversion. Additionally, observations of brain volume changes over the whole study period included patients in the placebo and once a week groups who were switched to IFN $\beta$-1a three times a week following conversion to CDMS.

The results from the REFLEX trial support the observation of improvements in MRI outcomes in previous studies of subcutaneous IFN $\beta-1 \mathrm{a}$ in patients with relapsing-remitting MS. ${ }^{27-29}$ Randomisation to a higher dose of subcutaneous IFN $\beta-1 \mathrm{a}$ (44 $\mu \mathrm{g}$ three times a week) has been associated with better MRI outcomes than randomisation to a lower dose $(22 \mu \mathrm{g}$ three times a week $)^{30}$ or to a lower, less frequent dose of intramuscular IFN $\beta-1 a{ }^{31}$

The limitations of the REFLEX trial have been discussed previously. ${ }^{8}$ Key questions are whether the short-term benefits observed on MRI outcomes will be maintained in the long term and whether early effects on MRI outcomes relate to later clinical outcomes. The extension to the REFLEX trial will provide additional insight into the long-term effects of the three times a week and once a week dosing regimens of IFN $\beta-1 \mathrm{a}, 44 \mu \mathrm{g}$ subcutaneously; ${ }^{32}$ however, previous studies suggest that MRI outcomes are useful measures of later clinical outcomes and that IFN $\beta$ therapy is likely to maintain. Longer-term studies in patients with relapsing MS or CIS have found that the benefits of IFN $\beta$ are maintained beyond 2 years. ${ }^{19}{ }^{32}$ Also, MRI outcomes after short-term therapy with IFN $\beta$ were predictive of both relapses at later time points ${ }^{33}$ and disability (in combination with the number of relapses). ${ }^{34}$

A growing body of evidence suggests that earlier IFN $\beta$ treatment of patients with MS or with CIS is associated with improved clinical and paraclinical outcomes compared with delayed treatment. ${ }^{18} 1932$ The REFLEX trial demonstrated that early subcutaneous IFN $\beta$ - 1 a treatment delayed the time to CDMS, with additional benefit of the higher-dose regimen over the lower-dose regimen. ${ }^{8}$ This secondary analysis of REFLEX found improved MRI outcomes in patients treated with both dosing regimens compared with placebo, and also found additional benefit of the higher-dose regimen. Together, these data support the rationale for early subcutaneous IFN $\beta$-1a treatment of patients with CIS suggestive of MS; however, these potential benefits of early treatment have to be balanced against the risk of treatment.

Acknowledgements The authors thank Paul Wilmott of Caudex Medical, Oxford, UK, for editorial assistance (supported by Merck Serono SA Geneva, Switzerland, a subsidiary of Merck KGaA, Darmstadt, Germany).

Contributors NDS, GC, LK, MSF: drafting/revising manuscript for content, including medical writing for content; study concept or design; analysis or interpretation of data; acquisition of data; study supervision or coordination. CHP, BMJU and LL: drafting/revising manuscript for content, including medical writing for content; analysis or interpretation of data. BH: drafting/revising manuscript for content, including medical writing for content; statistical analysis. FC-S: drafting/ revising manuscript for content, including medical writing for content; study concept or design; analysis or interpretation of data; statistical analysis. BS: drafting/revising manuscript for content, including medical writing for content; study concept or design; analysis or interpretation of data. DLJ: drafting/revising manuscript for content, including medical writing for content. FB: drafting/revising manuscript for content, including medical writing for content; analysis or interpretation of data; statistical analysis.

Funding Merck Serono SA Geneva, Switzerland, a subsidiary of Merck KGaA, Darmstadt, Germany, which was involved in the acquisition of data, statistical analysis (conducted by Florence Casset-Semanaz and Brian Hennessy, who were employees of Merck Serono SA Geneva, Switzerland, at the time of the study), study supervision and approval of data.

Competing interests NDS has received honoraria from Schering, Biogen Idec, Teva, Novartis and Merck Serono for consulting services, speaking and travel 
support; and serves on advisory boards for Merck Serono and Novartis. GC has received honoraria and consultation fees from Serono Symposia International Foundation, Merck Serono, Novartis, Teva Pharmaceutical Industries, Sanofi-Aventis, Bayer Schering and Biogen Dompè. LK has received institutional research support from Acorda, Actelion, Advancell, Allozyne, Barofold, Bayer, Bayhill, Biogen Idec, BioMarin, Boehringer Ingelheim, CSL Behring, Eli Lilly, Geneuro, Genmab, GlaxoSmithKline, Glenmark, Merck Serono, MediciNova, Novartis, Sanofi-Aventis, Santhera, Shire, Roche, Teva, UCB and Wyeth. MSF has received personal compensation from Bayer HealthCare, Biogen Idec, EMD Serono (Canada), Genzyme, Novartis, Opexa, Sanofi-Aventis and Teva Canada Innovation. CHP has received honoraria/consultation fees/research support from Actelion, Biogen Idec, Bayer Schering, GlaxoSmithKline, Merck Serono, Novartis, Teva, UCB and Roche. BMJU has received consultation fees from Biogen Idec, Novartis, Merck Serono, Synthon and Danone Research. BH, FC-S, LL, and BS were employees of Merck Serono SA Geneva, Switzerland, a subsidiary of Merck KGaA, Darmstadt, Germany, at the time of the study. DLJ is an employee of Caudex Medical, Oxford, UK and supported by Merck Serono SA Geneva, Switzerland, a subsidiary of Merck KGaA, Darmstadt, Germany. FB has received honoraria/consultation fees from Bayer-Schering, Biogen Idec, Merck Serono, Teva, Novartis, Roche, Sanofi-Aventis and Serono Symposia International Foundation.

Ethics approval The institutional review boards of all participating centres approved the study protocol.

Provenance and peer review Not commissioned; externally peer reviewed.

Data sharing statement Unpublished data are available with permission of the sponsor on application in writing to the corresponding author.

Open Access This is an Open Access article distributed in accordance with the Creative Commons Attribution Non Commercial (CC BY-NC 3.0) license, which permits others to distribute, remix, adapt, build upon this work non-commercially, and license their derivative works on different terms, provided the original work is properly cited and the use is non-commercial. See: http://creativecommons.org/ licenses/by-nc/3.0/

\section{REFERENCES}

1 Miller $D$, Barkhof $F$, Montalban $X$, et al. Clinically isolated syndromes suggestive of multiple sclerosis, part 1: natural history, pathogenesis, diagnosis, and prognosis. Lancet Neurol 2005;4:281-8.

2 Weinshenker BG, Bass B, Rice GP, et al. The natural history of multiple sclerosis: a geographically based study. 1. Clinical course and disability. Brain 1989; 112(Pt 1):133-46.

3 Confavreux C, Vukusic S, Moreau T, et al. Relapses and progression of disability in multiple sclerosis. N Engl J Med 2000;343:1430-8.

4 Comi G, Martinelli V, Rodegher M, et al. Effect of glatiramer acetate on conversion to clinically definite multiple sclerosis in patients with clinically isolated syndrome (PreCISe study): a randomised, double-blind, placebo-controlled trial. Lancet 2009;374:1503-11.

5 Jacobs LD, Beck RW, Simon JH, et al. Intramuscular interferon beta-1a therapy initiated during a first demyelinating event in multiple sclerosis. CHAMPS Study Group. N Engl J Med 2000;343:898-904.

6 Kappos L, Freedman MS, Polman CH, et al. Effect of early versus delayed interferon beta- $1 \mathrm{~b}$ treatment on disability after a first clinical event suggestive of multiple sclerosis: a 3-year follow-up analysis of the BENEFIT study. Lancet 2007;370:389-97.

7 Comi G, Filippi M, Barkhof $F$, et al. Effect of early interferon treatment on conversion to definite multiple sclerosis: a randomised study. Lancet 2001:357:1576-82.

8 Comi G, De Stefano N, Freedman MS, et al. Comparison of two dosing frequencies of subcutaneous interferon beta-1a in patients with a first clinical demyelinating event suggestive of multiple sclerosis (REFLEX): a phase 3 randomised controlled trial. Lancet Neurol 2012;11:33-41.

9 Polman $\mathrm{CH}$, Reingold SC, Edan G, et al. Diagnostic criteria for multiple sclerosis: 2005 revisions to the "McDonald Criteria". Ann Neurol 2005;58:840-6.

10 McDonald WI, Compston A, Edan G, et al. Recommended diagnostic criteria for multiple sclerosis: guidelines from the International Panel on the Diagnosis of Multiple Sclerosis. Ann Neurol 2001;50:121-7.

11 Polman CH, Reingold SC, Banwell B, et al. Diagnostic criteria for multiple sclerosis: 2010 revisions to the "McDonald criteria". Ann Neurol 2011;69:292-302.
12 Sormani MP, Bonzano L, Roccatagliata L, et al. Magnetic resonance imaging as a potential surrogate for relapses in multiple sclerosis: a meta-analytic approach. Ann Neurol 2009:65:268-75.

13 Smith ME, Stone LA, Albert PS, et al. Clinical worsening in multiple sclerosis is associated with increased frequency and area of gadopentetate dimeglumineenhancing magnetic resonance imaging lesions. Ann Neurol 1993;33:480-9.

14 Smith SM, Zhang Y, Jenkinson M, et al. Accurate, robust, and automated longitudinal and cross-sectional brain change analysis. Neuroimage 2002;17:479-89.

15 Barkhof $\mathrm{F}$, Polman $\mathrm{CH}$, Radue EW, et al. Magnetic resonance imaging effects of interferon beta-1b in the BENEFIT study: integrated 2-year results. Arch Neurol 2007:64:1292-8.

16 Beck RW, Chandler DL, Cole SR, et al. Interferon beta-1a for early multiple sclerosis: CHAMPS trial subgroup analyses. Ann Neurol 2002;51:481-90.

17 Jacobs LD, Cookfair DL, Rudick RA, et al. Intramuscular interferon beta-1a for disease progression in relapsing multiple sclerosis. Ann Neurol 1996;39:285-94.

18 Kinkel RP, Kollman C, O'Connor P, et al. IM interferon beta-1a delays definite multiple sclerosis 5 years after a first demyelinating event. Neurology 2006;66:678-84.

19 Kappos L, Freedman MS, Polman CH, et al. Long-term effect of early treatment with interferon beta-1b after a first clinical event suggestive of multiple sclerosis: 5 -year active treatment extension of the phase 3 BENEFIT trial. Lancet Neurol 2009:8:987-97.

20 Polman C, Kappos L, Freedman MS, et al. Subgroups of the BENEFIT study: risk of developing MS and treatment effect of interferon beta-1b. J Neurol 2008;255:480-7.

21 Frohman EM, Goodin DS, Calabresi PA, et al. The utility of MRI in suspected MS: report of the Therapeutics and Technology Assessment Subcommittee of the American Academy of Neurology. Neurology 2003;61:602-11.

22 Korteweg T, Tintore M, Uitdehaag B, et al. MRI criteria for dissemination in space in patients with clinically isolated syndromes: a multicentre follow-up study. Lancet Neurol 2006;5:221-7.

23 Di Filippo M, Anderson VM, Altmann DR, et al. Brain atrophy and lesion load measures over 1 year relate to clinical status after 6 years in patients with clinically isolated syndromes. J Neurol Neurosurg Psychiatry 2010;81:204-8.

24 Traboulsee A, Zhao G, Li DK. Neuroimaging in multiple sclerosis. Neurol Clin 2005;23:131-48, vii.

25 Filippi $M$, Rovaris $M$, Inglese $M$, et al. Interferon beta-1a for brain tissue loss in patients at presentation with syndromes suggestive of multiple sclerosis: a randomised, double-blind, placebo-controlled trial. Lancet 2004;364:1489-96.

26 Zivadinov R, Reder AT, Filippi M, et al. Mechanisms of action of disease-modifying agents and brain volume changes in multiple sclerosis. Neurology 2008;71:136-44.

27 Li DK, Paty DW, UBC MS/MRI Analysis Research Group, PRISMS Study Group. Magnetic resonance imaging results of the PRISMS trial: a randomized, double-blind, placebo-controlled study of interferon-beta1a in relapsing-remitting multiple sclerosis. Ann Neurol 1999;46:197-206.

28 PRISMS Study Group. Randomised double-blind placebo-controlled study of interferon beta-1a in relapsing/remitting multiple sclerosis. Lancet 1998:352:1498-504.

29 De Stefano N, Curtin F, Stubinski B, et al. Rapid benefits of a new formulation of subcutaneous interferon beta-1a in relapsing-remitting multiple sclerosis. Mult Scler 2010;16:888-92.

30 PRISMS Study Group, University of British Columbia MS/MRI Analysis Group. PRISMS-4: long-term efficacy of interferon-beta-1a in relapsing MS. Neurology 2001;56:1628-36.

31 Schwid SR, Panitch HS. Full results of the Evidence of Interferon Dose-Response-European North American Comparative Efficacy (EVIDENCE) study: a multicenter, randomized, assessor-blinded comparison of low-dose weekly versus high-dose, high-frequency interferon beta-1a for relapsing multiple sclerosis. Clin Ther 2007;29:2031-48.

32 Kappos L, Traboulsee A, Constantinescu C, et al. Long-term subcutaneous interferon beta-1a therapy in patients with relapsing-remitting MS. Neurology 2006:67:944-53.

33 Sormani MP, Stubinski B, Cornelisse P, et al. Magnetic resonance active lesions as individual-level surrogate for relapses in multiple sclerosis. Mult Scler J 2011:17:541-9.

34 Sormani MP, Li DK, Bruzzi P, et al. Combined MRI lesions and relapses as a surrogate for disability in multiple sclerosis. Neurology 2011;77:1684-90. 